ADDENDUM

doi:10.1038/nature10395

\title{
The thermodynamic meaning of negative entropy
}

Lídia del Rio, Johan Åberg, Renato Renner, Oscar Dahlsten

\& Vlatko Vedral

Nature 474, 61-63 (2011)

To clarify the implications of our result, we note that, although the erasure processes we considered in our Letter can have negative work cost (that is, they can yield work), they do not violate the second law of thermodynamics, because they are not cyclic processes. A negative work cost is associated with the consumption of entanglement, which can only be restored by doing work. Our results are also consistent with the original unconditional form of Landauer's principle, which says that if there is no information available about the data being erased, the cost of erasure is always positive. Similarly, because in a computation with deterministic classical output the joint entropy of all registers conditioned on the output cannot be negative, the overall work cost of such a computation is always positive or zero (even though temporary quantum correlations may be created and exploited during the course of the computation). In fact, standard techniques of reversible information processing allow any deterministic classical algorithm to be performed, on a classical or quantum computer, in a thermodynamically reversible fashion, with work cost arbitrarily close to zero ${ }^{1,2}$. These clarifications are developed in more detail in the Supplementary Information to this Addendum. We thank Charles H. Bennett for remarks on reversible computation.

Supplementary Information is linked to the online version of the Addendum at www.nature.com/nature.

1. Bennett, C. H. The thermodynamics of computation-a review. Int. J. Theor. Phys. 21, 905-940 (1982).

2. Watrous, J. Quantum computational complexity. In Encyclopaedia of Complexity and Systems Science (ed. Meyers, R. A.) Part 17, 7174-7201 (Springer, 2009). 